\title{
Palladium Catalyzed Enantioselective Hayashi-Miyaura Reaction for Pharmaceutically Important 4-Aryl-3,4-Dihydrocoumarins
}

\author{
Jixing Lai, $\uparrow$ Chen Yang, $\uparrow$ René Csuk, † Baoan Song, $\uparrow$ Shengkun Li $*, \uparrow$
}

$†$ Laboratory Breeding Base of Green Pesticide and Agricultural Bioengineering, Key Laboratory of Green Pesticide and Agricultural Bioengineering, Ministry of Education, Guizhou University, Huaxi District, Guiyang 550025, China

†Organic Chemistry, Martin-Luther-University Halle-Wittenberg, Kurt-Mothes-Str. 2, D-06120 Halle (Saale), Germany

Supporting Information Placeholder

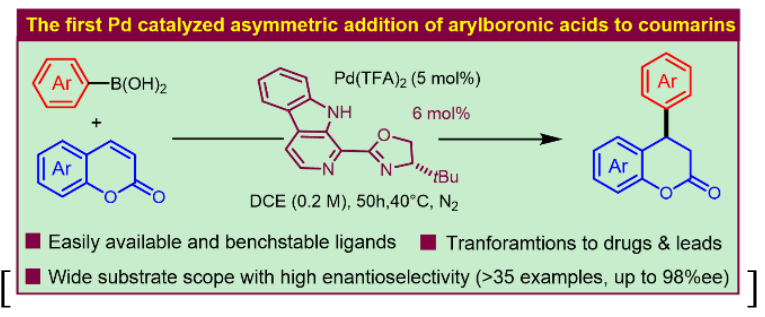

The first palladium-catalyzed asymmetric addition of arylboronic acids to coumarins was successfully established, providing a straightforward asymmetric approach to achieving pharmaceutically important 4-aryl-3,4-dihydrocoumarins. This methodology features easily accessible and bench-stable ligands, a wide substrate scope, mild conditions and good accommodation of challenging, electron-withdrawing arylboronic acids.

Coumarins are pharmaceutically important characteristics in numerous bioactive natural products and synthesized drug leads, holding various activities ranging from anti-inflammatory, antioxidant, antimicrobial, to immunomodulatory properties. ${ }^{1}$ Among different analogs, 4-aryl-3,4-dihydrocoumarins have attracted increasing attention from both synthetic communities and medicinal unions (Figure 1). Notably, dihydrocoumarins $\mathbf{1}$ and $\mathbf{2}$ showed outstanding anti-inflammatory and antioxidant activities. ${ }^{\text {la }}$ These coumarins can always serve as synthetic hubs for the construction of pharmaceutically important derivatives, exemplified by the GPR40 agonist 3,3-diarylpropanoic acid $\mathbf{3}^{2}$ and the novel ROR $\gamma$ antagonists diphenylpropanamide ML209 ${ }^{3}$ and muscarinic antagonist $(R)$-tolterodine ${ }^{4}$. The pre-validated chiral differentiation of the biological effects ${ }^{3}$ of 4-aryl-3,4-dihydrocoumarins and derivatives protruded the corresponding asymmetric synthesis as an important research topic.

Prominent advances in the asymmetric synthesis of 4-aryl-3,4dihydrocoumarins (Figure $2 \mathrm{~A})^{5}$ have been achieved through (1) hydroesterification of alkenylphenols $4,{ }^{6}(2)$ asymmetric reduction of coumarins $5,{ }^{7}$ (3) Baeyer-Villiger oxidation of indan-1-ones $6{ }^{8}$ (4) addition of 1,3-dicarbonyl compounds to $o$-quinone methides generated in situ from arylsulfonyl-alkylphenols $7,{ }^{9}$ and (5) the addition of arylboronic acids to coumarins (Figure 2B). This privileged scaffold can also be prepared from enals $\mathbf{8}$ and phenols 9 through carbene-catalyzed annulation ${ }^{10}$ or from para-quinone methides 10 via Rauhut-Currier reaction ${ }^{11}$ (Figure 2A). Recently, Tang developed an elegant palladium/Wingphos-catalyzed asymmetric hydroesterification of diarylmethyl carbinols $\mathbf{1 1}$ to furnish chiral 4-aryl-3,4-dihydrocoumarins ${ }^{12}$. In the continuing interest in the asymmetric addition of arylboronic acids to $\alpha, \beta$-unsaturated compounds $^{13}$ (Hayashi-Miyaura reactions), we envisage the asymmetric paradigm for the addition of arylboronic acids $\mathbf{1 2}$ to coumarins $\mathbf{1 3}$ is appealing given the versatility and facile availability of both reaction partners. Success in this will not only provide an alternative approach to the aforementioned bioactive products but also expand of the chemical space of complicated chiral dihydrocoumarins.

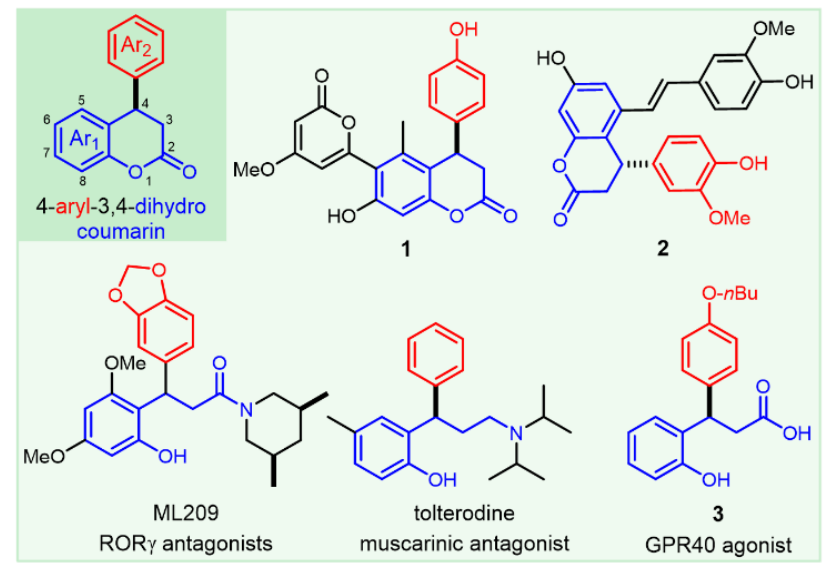

Figure 1. 4-Aryl-3,4-dihydrocoumarins relevant molecules

From a historical point of view, asymmetric addition of arylboronic acids to coumarins has mainly been realized by rhodium catalysis which was characterized by the development of various chiral biphosphorus ligands (Figure 2B-1). These advances were mainly showcased by the utilization of $(R)$-Segphos, ${ }^{14}\left(\mathrm{MeO}-\mathrm{F}_{12}-\right.$ BIPHEP), ${ }^{15}$ modified BIPHEP $\mathbf{1 4}^{16}$, (S)-BICMAP catalysts, ${ }^{17}$ Chiraphos $^{18}$ and the air-sensitive phospholane phosphite ligand $(R$, $R, R$ )-BOBPHOS from multistep synthesis. ${ }^{19}$ Additionally, some 
chiral diene ligands, such as ligand $\mathbf{1 5}^{20}$ and ligand $\mathbf{1 6}^{21}$ were also applied specifically.

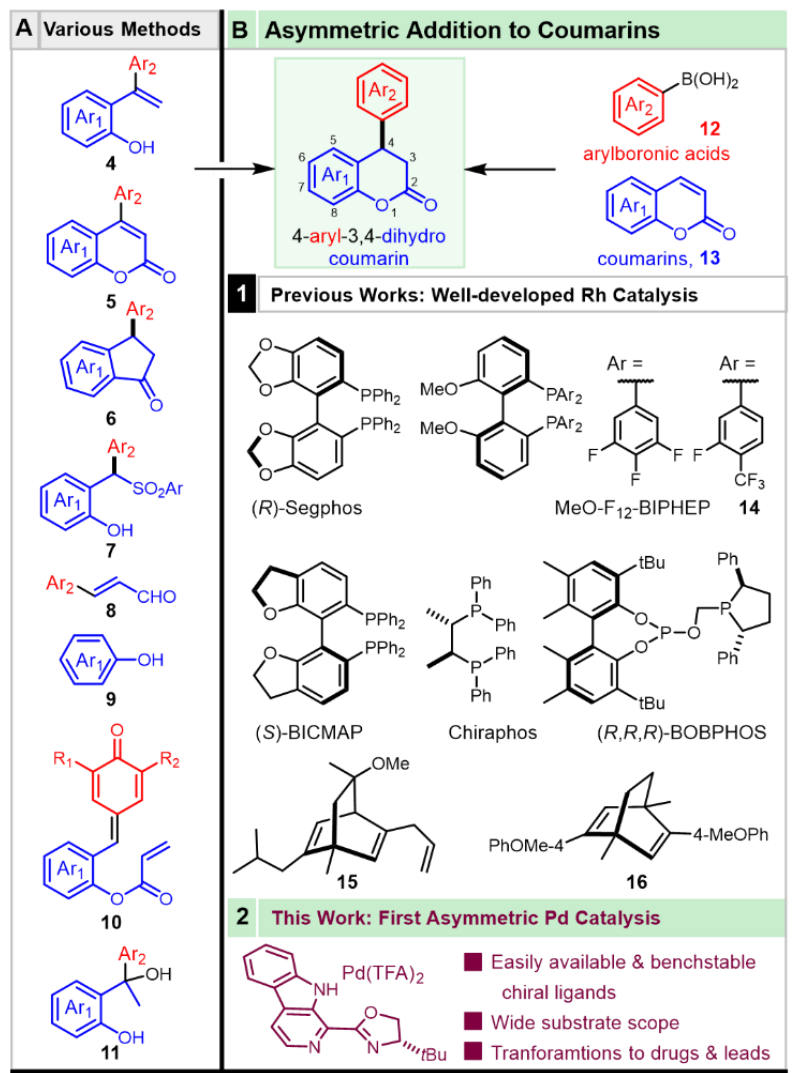

Figure 2. Various methods for 4-aryl-3,4-dihydrocoumarins

Notwithstanding some promising achievements, the aforementioned rhodium catalytic approaches encountered many challenges in view of synthetic feasibility and economy and the compatibility of electron-donating coumarins and electron-deficient or ringfused arylboronic acids. Therefore, the discovery of novel catalytic systems with operational simplicity and facile accessibility is highly desirable for addressing these problematic issues. Our hypothesis was intrigued by the only example of the $\mathrm{Pd} /$ bipy system for the synthesis of diphenylpropanamides as ROR $\gamma$ antagonists ${ }^{3}$. It is noteworthy that this system was not tailored to the addition to coumarins but was applied immediately by just a horizontal transfer from that for $\alpha, \beta$-unsaturated ketones and esters ${ }^{22}$. Palladiumcatalyzed asymmetric addition is yet an untouched area and we envision the discovery of suitable catalysts is the key to overcoming this issue. Herein, we would like to document the first palladium catalytic system for the enantioselective addition of a large range of arylboronic acids to various coumarins (Figure 2B2). This protocol employs air-stable and easily accessible chiral ligands and can accommodate electron-poor arylboronic acids.

The identification of suitable chiral $N$-containing ligands commenced first for the Pd-catalyzed enantioselective addition of arylboronic acid 12a to coumarin 13a (Scheme 1A). Easily accessible PyOx-type ligands, including $t \mathrm{Bu}-\mathrm{PyOx}$, NL1 and NL2, were initially investigated. The commonly used $t \mathrm{Bu}-\mathrm{PyOx}$ can facilitate this transformation by delivering the desired compound 14a in $42 \%$ enantiomeric excess (ee) with a modest isolated yield. The tiny increment of the steric hindrance near the coordinating position is not tolerant, as the 6-methyl- $t$ Bu-PyOx NL2 did not provide any detectable 14a. Since ring-fused pyridine-oxazolines always provide new solutions for unprecedented transformations, we tested a large variation of (hetero)aryl fused pyridineoxazoline ligands (NL3-NL15). Both quinoline-oxazoline NL3 and $\alpha$-phenyl $\beta^{3}$-Carox NL6 were inert to this addition, which may be due to the increased steric hindrance around the catalytic center. The isoquinoline-oxazolines NL4 and NL7, slightly increased the production of 14a at the cost of enantioselectivity. The introduction of an indole (NL5) or a pyrrole (NL8) backbone improved either the yield or the enantioselectivity of dihydrocoumarin 14a. Gratifyingly, the orthogonal modification led to an attempt at using $\mathrm{t}$-Bu- $\beta^{3}$-Carox NL9, which allowed a significant improvement of the ee value to $78 \%$ ee.

Scheme 1. Optimization of the asymmetric addition of arylboronic acid $\mathbf{1 a}$ to coumarin $\mathbf{2 a}$

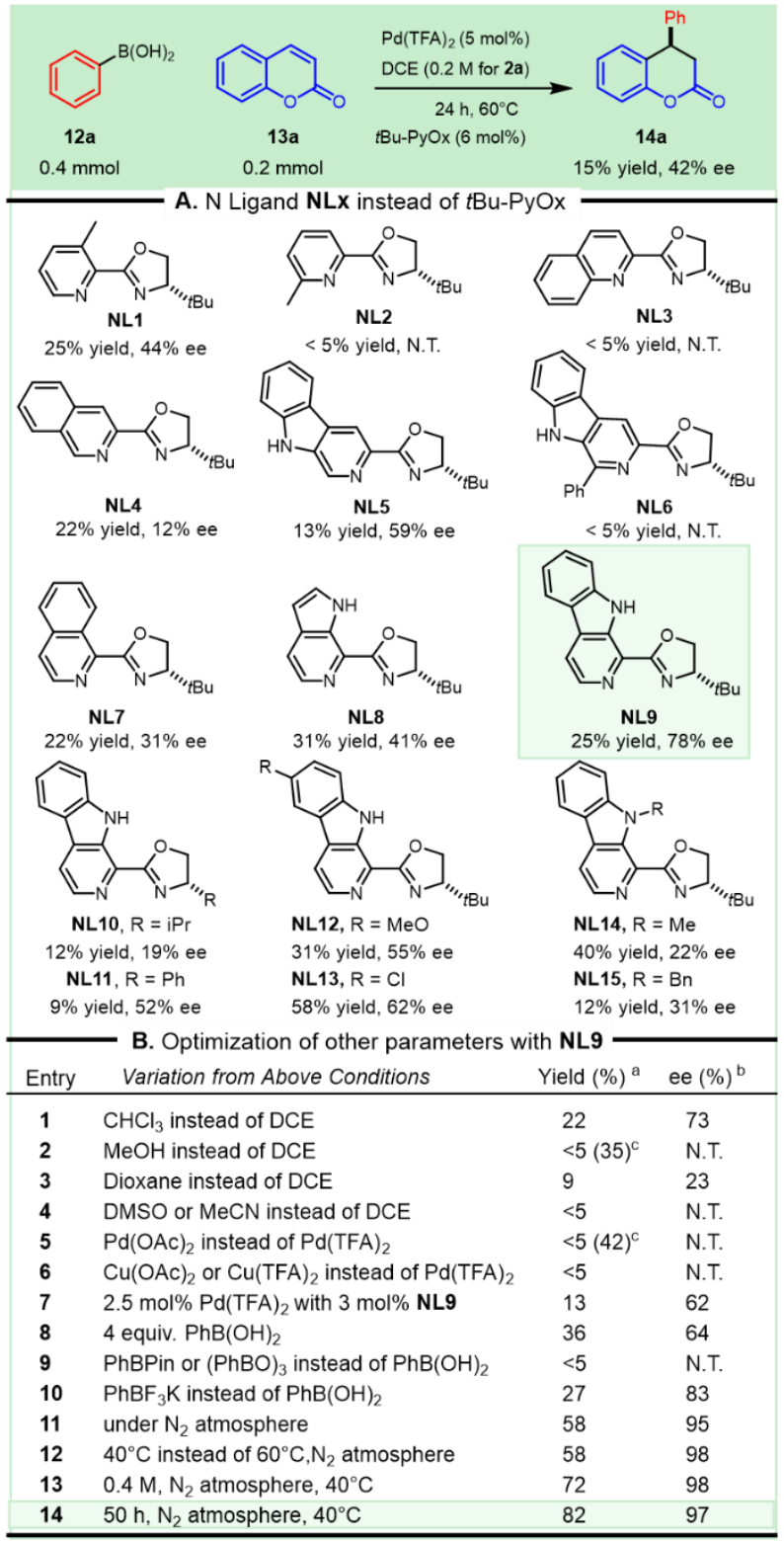

Note ${ }^{\mathrm{a}}$, isolated yield; ${ }^{\mathrm{b}}$, detected by chiral HPLC on a chiralcel OD-H column (the absolute configuration was assigned by comparison with the literature); ${ }^{\mathrm{c}}$, the data in parentheses are the isolated yields of 4-Ph-coumarin

Tuning the steric factors on the oxazoline ring (NL10 and NL11) or the electronic properties in the indole ring (NL12, NL13, NL14, and NL15) turned out to be inefficient. The other parameters (Scheme 1B) were subsequently optimized with the chiral ligand NL9. The variation of the reaction media revealed that the chlorinated polar solvent can provide a comparable result (entry 1), while DMSO or MeCN did not furnish detectable dihy- 
drocoumarin 14a (entry 4). The polar protic solvent methanol provided the Heck-type product 4-ph-coumarin in 35\% yield (entry 2). A similar outcome was observed when the counterion was changed to $\mathrm{CH}_{3} \mathrm{COO}^{-}$(entry 5). Variation of the central metal to copper (entry 6 ) or a decrease in the catalyst loading (entry 7) was detrimental. Increasing the loading of $\mathrm{PhB}(\mathrm{OH})_{2}$ can slightly improve the yield of $\mathbf{1 4 a}$, while a sharp erosion of the enantioselectivity was observed (entry 8). Dihydrocoumarin 14a was also harvested by directly using $\mathrm{PhBF}_{3} \mathrm{~K}$ (entry 10 ), while $\mathrm{PhBPin}$ or $(\mathrm{PhBO})_{3}$ was demonstrated to be inefficient (entry 9). Fruitful improvement was achieved by simply conducting the experiment under a $\mathrm{N}_{2}$ atmosphere, in which the yield and enantioselectivity of 14a were promoted to $58 \%$ and $95 \%$, respectively (entry 11). The enantioselectivity was enhanced when the temperature was lowered to $40{ }^{\circ} \mathrm{C}$ without scarification of the conversion (entry 12). The conversion can be improved in a condensed solution (entry 13) or a prolonged process (entry 14). The optimal conditions, as shown in entry 14 , provided 4-aryl-3,4-dihydrocoumarin 14a in $82 \%$ yield and $97 \%$ ee.

Scheme 2. Substrate scope with respect to the coumarins a

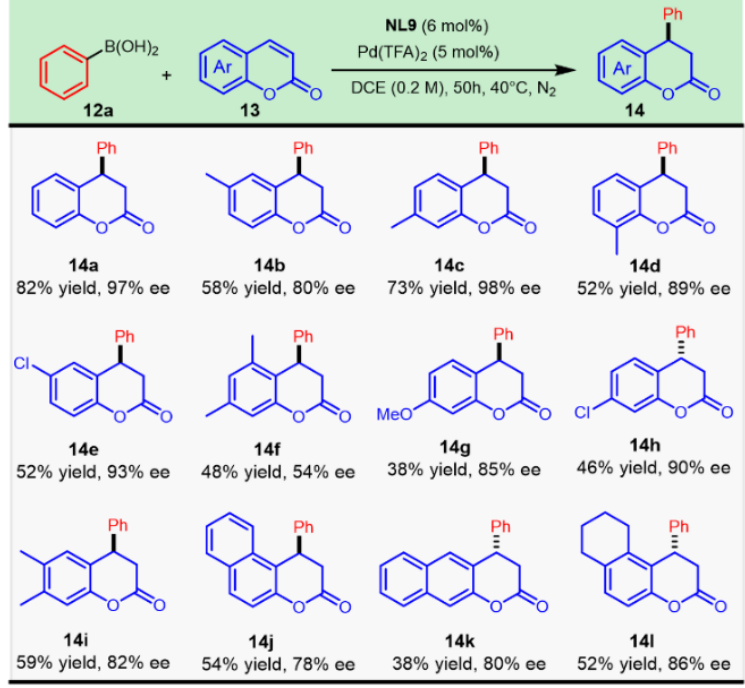

Note $^{\text {a }}$, All the yields refer to the isolated yield. The ee values were determined by HPLC on a chiral phase.

The optimized protocol has already proven to be capable of coupling the commercially available arylboronic acid 12a with a variety of coumarins 13 (Scheme 2). Delightfully, different decorations with either electron-withdrawing or electron-donating groups on the aromatic segment could proceed smoothly to afford 4-phenyl-3,4-dihydrocoumarins with a good to an excellent stereocontrol. It is noteworthy that the dependence of the enantioselectivities on the substituted positions was observed. Migration of the methyl group from the 6-position to the 7-position or 8-position improved the enantioselectivity from $80 \%$ ee (14b) to $98 \%$ ee (14c) and $89 \%$ ee (14d), respectively. The problematic electrondonating coumarin in the rhodium catalysis ${ }^{14}$ could also be converted with good enantioselectivity of $85 \%$ ee $(\mathbf{1 4 g})$. Shifting the chloro-group did not significantly affect the enantioselectivity and yields (14e and 14h). Additional decoration of the methyl group to either the 5-position (14f) or 6-position (14i) demonstrated a deleterious effect on both productivity and selectivity compared with product 14c. Interestingly, this method also promoted the ring-fused coumarins to react smoothly with phenylboronic acid 12a. The fused position showed a negligible effect on the stereocontrol $(\mathbf{1 4} \mathbf{j}$ vs. $\mathbf{1 4 k})$, while the [5,6]-fused isomer $\mathbf{1 4} \mathbf{j}$ gave a more satisfying yield.

Our attention was turned to exploring the scope of the nucleophilic arylboronic acids 12 (Scheme 3). Delightfully, a wide varie- ty of chiral 4-aryl-3,4-dihydrocoumarins could be achieved with good to excellent enantioselectivity. The generality was corroborated by the successful utilization of arylboronic acids with various functional groups, including ethers, thioether, halogens, fused rings and heterocycles. Although transferring the methyl group from the para or meta position to the ortho position impaired the reactivity (15a and $\mathbf{1 5 b}$ vs. 15c), slightly better enantioselectivity was detected due to the increased steric hindrance.

The similar variation of halogenated arylboronic acids showed a more sensitive effect on the enantioselectivity, exemplified by the preparation of compounds $15 \mathbf{k}$ and 15l. Notably, paramethylthio phenylboronic acid, which has a strong coordinating ability, did not impair either the reactivity or the enantioselectivity, providing $15 \mathrm{~g}$ in $67 \%$ isolated yield with up to $96 \%$ ee. The trimethylsilyl group was well tolerated, realizing the enantioselective synthesis of $\mathbf{1 5 h}$ with a satisfactory yield. The challenging naphthylboronic acids in $\mathrm{Rh}$ catalysis, ${ }^{17 \mathrm{a}}$ were successfully coupled with coumarin 13a to deliver $\mathbf{1 5 n}$ and $\mathbf{1 5 o}$ in $92 \%$ ee and $88 \%$ ee, respectively. However, a limitation was observed when the 2,3disubstituted coumarin (15t) or ortho-chloro-phenylboronic acid $(\mathbf{1 5} \mathbf{u})$ was recruited. The reaction was problematic by employing either aliphatic boronic acid (15v) or 3-thienyl boronic acid (15w).

Scheme 3. Scope with respect to the arylboronic acids a
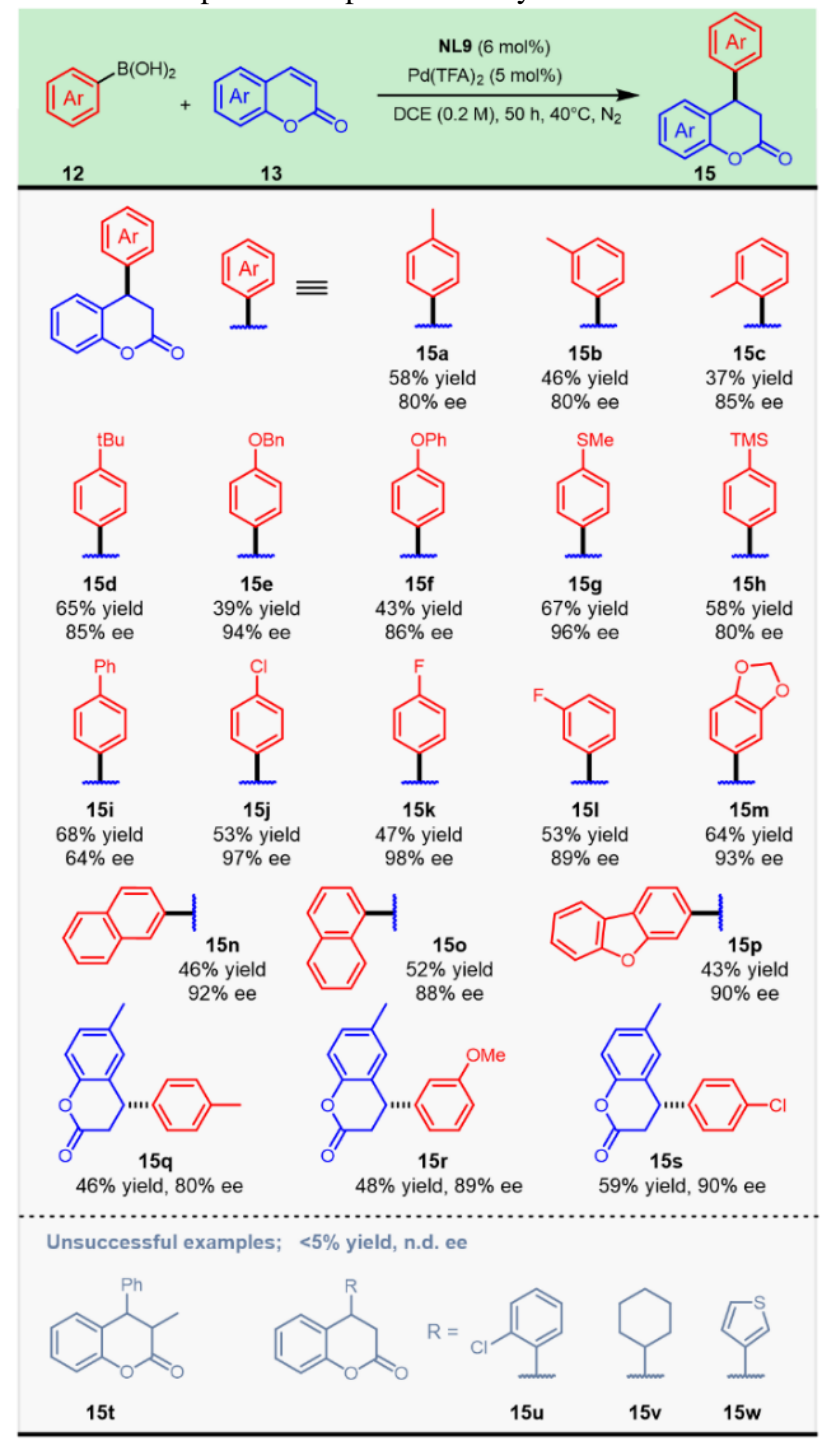

Note $^{\mathrm{a}}$, as in Scheme 2. 
This method is anticipated to be of value for the asymmetric coupling of the electron-deficient arylboronic acids, which were challenging substrates in the previous $\mathrm{Rh}$ catalysis. Invigorated by the prevalidated examples $(\mathbf{1 5} \mathbf{j}, \mathbf{1 5} \mathbf{k}, \mathbf{1 5 l}$ and $\mathbf{1 5 s})$ shown in Scheme 3 , the compatibility of the current chemistry was further verified by enantioselective coupling fluoro-phenylboronic acids with various coumarins (Figure 3). Notably, 4-aryl-3,4dihydrocoumarins $\mathbf{1 6 e}$ and $\mathbf{1 6 f}$ can be furnished in appreciable results in view of both yield and enantioselectivity, which contained unfavorable factors from both reaction partners. ${ }^{14,17 \mathrm{a}}$

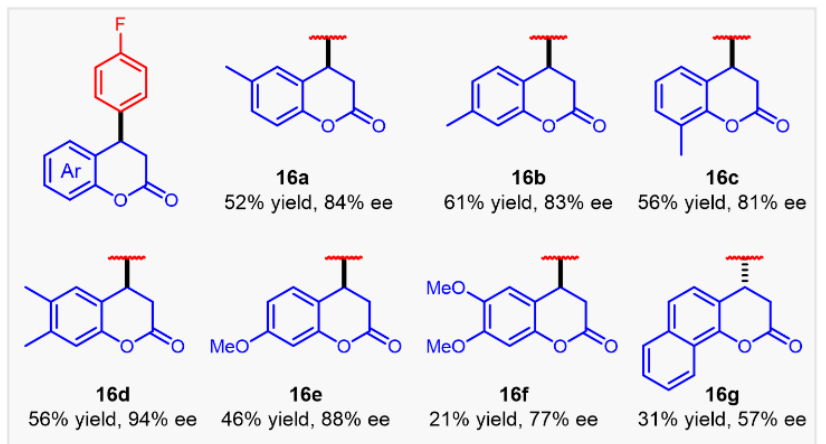

Figure 3. Asymmetric addition of $p$-fluoro-phenylboronic acid

Compared with the previous $\mathrm{Pd} /$ chiral amine co-catalysis for the enantioselective synthesis of 4-phenylchroman-2-one $\mathbf{1 4 a},{ }^{23}$ the current methodology represented a straightforward, convenient and higher enantioselective tactic (Scheme 4A). The current transformation will facilitate an alternative approach to the enantioselective synthesis of the urological drug $(R)$-tolterodine ${ }^{14}$ or its isomers from 4-aryl-3,4-dihydrocoumarins $14 b, \mathbf{1 4 c}$ and $\mathbf{1 4 d}$ (Scheme 4B). Additionally, compounds 15e, 15f, and $15 \mathrm{~g}$ (Scheme 3) could be transformed ${ }^{12}$ to the enantioenriched $\beta, \beta$ diaryl carboxylic acids as analogs of potent GPR40 agonists for the treatment of diabetes. It is worth noting that aminolysis of the chiral 4-aryl-3,4-dihydrocoumarins with secondary amines will provide a modular and expeditious construction of a plethora of diphenylpropanamides, which have proven to be effective for treating $\mathrm{T}_{\mathrm{H}} 17$-related autoimmune diseases. ${ }^{24}$ The facile access to coumarins and the availability and variety of both arylboronic acids and amines may expand the chemical space of these pharmaceutically important amides.

Inspired by this late-stage amenability, we embarked on the aminolysis of 4-aryl-3,4-dihydrocoumarins to the corresponding amides, which can be deemed as the analogs of flumorph. The resulting enantioenriched amides 17a-17C (Scheme 4C) can be readily acquired without decreasing the optical purity of the dihydrocoumarin precursors, highlighting the synthetic potential of this tactic. The in vitro antifungal phenotypic test (see the Supporting Information for more details) showed that the inhibitory efficacy of coumarin improved with the addition of arylboronic acid (Scheme 4D, 13b vs. 14b), while the corresponding product 17b from aminolysis did not lead to further enhancement of antifungal potential. The resultant diphenylpropanamides demonstrated quite different antifungal potency from that of flumorph, showing that the unsaturated $\mathrm{C}=\mathrm{C}$ bond of flumorph may be a crucial factor in acquiring significant antifungal potential against phytophthora capsici.

In summary, the first palladium-catalyzed asymmetric addition of arylboronic acids to coumarins was successfully established. This convenient protocol employed air-stable and easily accessible chiral nitrogen-containing ligands and provided a straightforward asymmetric approach to achieving pharmaceutically important 4-aryl-3,4-dihydrocoumarins. The generality was demonstrated by a successful asymmetric addition of various arylboronic acids to a wealth of coumarins. Notably, this methodology fea- tures mild conditions and accommodation of the challenging arylboronic acids encountered in the established Rh catalysts. Further progress is ongoing toward the discovery of novel pharmaceutically important leads through the synergistic interaction of synthetic methodologies and biological exploration.

Scheme 4. Applications of the current methodology

A Comparison of different $\mathrm{Pd}$ catalysis for synthesis of $14 \mathrm{a}$

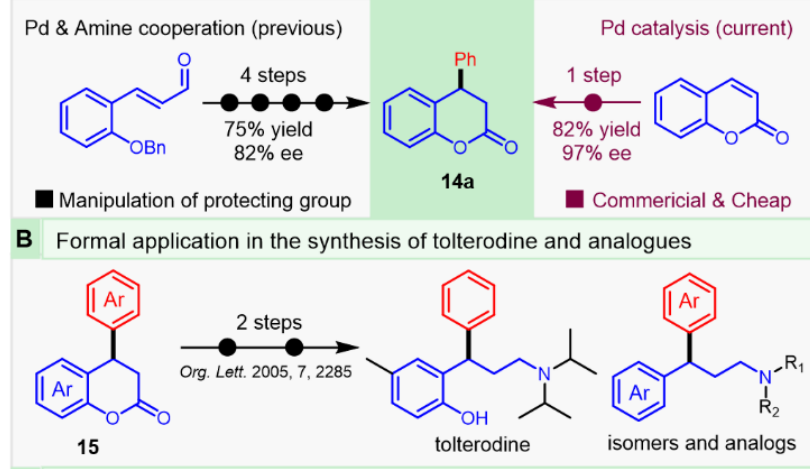

C Synthesis of chiral diphenylpropanamides (ROR $\gamma$ antagonists and analogs)

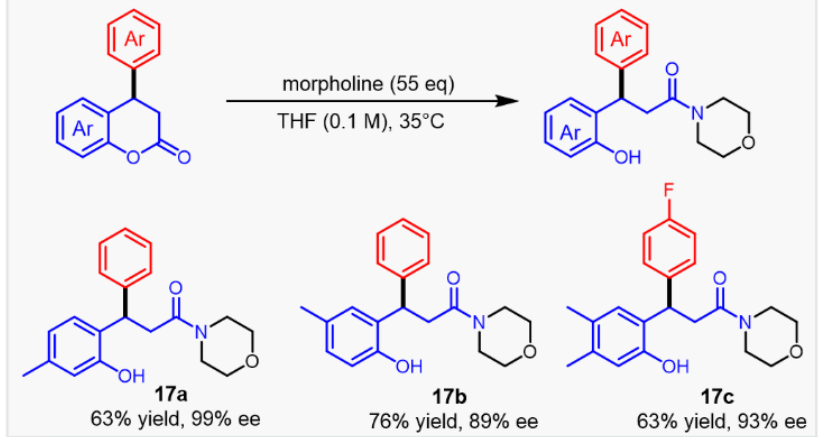

D Selected antifungal examples at $50 \mathrm{uM}$ (inhibitory rate, \%)

\begin{tabular}{cccccccc} 
Compd. & R.S. & S.S. & B.C. & F.G. & P.C. & P.O. & C.C. \\
\hline 13b & 0 & 0 & 17.3 & 0 & 7.4 & 28.6 & 16.3 \\
rac-14b & 45.4 & 15.1 & 30.9 & 13.7 & 17.9 & 27.6 & 16.3 \\
rac-17b & 0 & 3.8 & 29.4 & 1.2 & 34.7 & 27.6 & 12.8 \\
rac-17c & 0 & 1.9 & 21.8 & 15.7 & 10.5 & 31.6 & 14.0 \\
flumorph & 2.8 & 5.7 & 23.6 & 0 & 97.9 & 10.2 & 12.8 \\
\hline R.S. : Rhizoctonia solani, S.S. : Sclerotinia scleotiorum, B.C. : Botrytis cinerea, F.G.: Fusarium
\end{tabular}

graminearum, P.C. : Phytophthora capsici, P.O. : Pyricularia oryzae, C.C. : Colletotrichum capsici.

\section{ASSOCIATED CONTENT}

\section{Supporting Information}

The Supporting Information is available free of charge on the ACS Publications website.

Synthetic procedures, characteristic data, HPLC Traces, NMR spectra and antifungal data (file type, i.e., PDF)

\section{AUTHOR INFORMATION}

\section{Corresponding Author}

* Shengkun Li

E-mail: sk1505@outlook.com

ORCID: 0000-0001-5458-0811

\section{Author Contributions}

S. Li conceived and designed this work, J. Lai and C. Yang performed experiments and provided the results, S. Li and J. Lai analyzed the data and all authors wrote the manuscript.

Notes

The authors declare no competing financial interest.

\section{ACKNOWLEDGMENT}


This work was financially supported by the National Natural Science Foundation of China (21772094, 21977049), Research Foundation of Guizhou University (202016) and the Natural Science Foundation of Jiangsu Province (BK20191306).

\section{REFERENCES}

(1) (a) dos Santos, W. H.; da Silva-Filho, L. C., NbCl5promoted synthesis of 4-aryl-3,4-dihydrocoumarins by multicomponent reaction. Synthesis 2012, 44, 3361-3365; (b) Yu, D.; Suzuki, M.; Xie, L.; Morris-Natschke, S. L.; Lee, K.-H., Recent progress in the development of coumarin derivatives as potent antiHIV agents. Med. Res. Rev. 2003, 23, 322-345; (c) Gliszczyńska, A.; Brodelius, P. E., Sesquiterpene coumarins. Phytochem. Rev. 2012, 11, 77-96; (d) Zhang, K.; Ding, W.; Sun, J.; Zhang, B.; Lu, F.; Lai, R.; Zou, Y.; Yedid, G., Antioxidant and antitumor activities of 4arylcoumarins and 4-aryl-3,4-dihydrocoumarins. Biochimie 2014, 107, 203-210.

(2) Song, F.; Lu, S.; Gunnet, J.; Xu, J. Z.; Wines, P.; Proost, J.; Liang, Y.; Baumann, C.; Lenhard, J.; Murray, W. V.; Demarest, K. T.; Kuo, G.-H., Synthesis and Biological Evaluation of 3-Aryl-3-(4phenoxy)-propionic Acid as a Novel Series of G Protein-Coupled Receptor 40 Agonists. J. Med. Chem. 2007, 50, 2807-2817.

(3) Huh, J. R.; Englund, E. E.; Wang, H.; Huang, R.; Huang, P.; Rastinejad, F.; Inglese, J.; Austin, C. P.; Johnson, R. L.; Huang, W.; Littman, D. R., Identification of Potent and Selective Diphenylpropanamide ROR $\gamma$ Inhibitors. ACS Med. Chem. Lett. 2013, 4, 79-84.

(4) (a) Srinivas, K.; Srinivasan, N.; Reddy, K. S.; Ramakrishna, M.; Reddy, C. R.; Arunagiri, M.; Kumari, R. L.; Venkataraman, S.; Mathad, V. T., An Improved, Scalable, and Impurity-Free Process for Tolterodine Tartrate. Org. Process Res. Dev. 2005, 9, 314-318; (b) Castro, K. A. D.; Ko, J.; Park, D.; Park, S.; Rhee, H., Reduction of Ethyl Benzoylacetate and Selective Protection of 2-(3-Hydroxy-1phenylpropyl)-4-methylphenol: A New and Facile Synthesis of Tolterodine. Org. Process Res. Dev. 2007, 11, 918-921.

(5) Leitis, Z., Synthesis of enantiomerically enriched 4-aryl3,4-dihydrocoumarins (microreview). Chem. Heterocycl. Compd. ( $N$. Y., NY, U. S.) 2016, 52, 527-529.

(6) Li, J.; Chang, W.; Ren, W.; Liu, W.; Wang, H.; Shi, Y., A palladium-catalyzed enantioselective hydroesterification of alkenylphenols with phenyl formate. A facile approach to optically active dihydrocoumarins. Org. Biomol. Chem. 2015, 13, 10341-10347.

(7) (a) Barancelli, D. A.; Jr., A. G. S.; Taylor, J. G.; Correia, C. R. D., Coumarins from Free ortho-Hydroxy Cinnamates by HeckMatsuda Arylations: A Scalable Total Synthesis of (R)-Tolterodine. Org. Lett. 2012, 14, 6036-6039; (b) Gallagher, B. D.; Taft, B. R.; Lipshutz, B. H., Asymmetric Conjugate Reductions of Coumarins. A New Route to Tolterodine and Related Coumarin Derivatives. Org. Lett. 2009, 11, 5374-5377.

(8) Yue, G.; Lei, K.; Hirao, H.; Zhou, J. S., PalladiumCatalyzed Asymmetric Reductive Heck Reaction of Aryl Halides. Angew. Chem., Int. Ed. 2015, 54, 6531-6535.

(9) Caruana, L.; Mondatori, M.; Corti, V.; Morales, S.; Mazzanti, A.; Fochi, M.; Bernardi, L., Catalytic Asymmetric Addition of Meldrum's Acid, Malononitrile, and 1,3-Dicarbonyls to orthoQuinone Methides Generated In Situ Under Basic Conditions. Chem. -Eur. J. 2015, 21, 6037-6041.

(10) Li, G.-T.; Li, Z.-K.; Gu, Q.; You, S.-L., Asymmetric Synthesis of 4-Aryl-3,4-dihydrocoumarins by N-Heterocyclic Carbene Catalyzed Annulation of Phenols with Enals. Org. Lett. 2017, $19,1318-1321$.

(11) Zhang, X.-Z.; Gan, K.-J.; Liu, X.-X.; Deng, Y.-H.; Wang, F.-X.; Yu, K.-Y.; Zhang, J.; Fan, C.-A., Enantioselective Synthesis of
Functionalized 4-Aryl Hydrocoumarins and 4-Aryl Hydroquinolin-2ones via Intramolecular Vinylogous Rauhut-Currier Reaction of paraQuinone Methides. Org. Lett. 2017, 19, 3207-3210.

(12) Tian, D.; Xu, R.; Zhu, J.; Huang, J.; Dong, W.; Claverie, J.; Tang, W., Asymmetric Hydroesterification of Diarylmethyl Carbinols. Angew. Chem., Int. Ed. 2021, 60, 6305-6309.

(13) (a) Li, W.; Wang, G.; Lai, J.; Li, S., Multifunctional isoquinoline-oxazoline ligands of chemical and biological importance. Chem. Commun. 2019, 55, 5902-5905; (b) Lai, J.; Li, W.; Wei, S.; Li, S., Natural carbolines inspired the discovery of chiral CarOx ligands for asymmetric synthesis and antifungal leads. Org. Chem. Front. 2020, 7, 2263-2268.

(14) Chen, G.; Tokunaga, N.; Hayashi, T., Rhodium-Catalyzed Asymmetric 1,4-Addition of Arylboronic Acids to Coumarins: Asymmetric Synthesis of (R)-Tolterodine. Org. Lett. 2005, 7, 22852288.

(15) Korenaga, T.; Maenishi, R.; Osaki, K.; Sakai, T., Highly active rhodium catalyst with electron-poor diphosphine enables efficient synthesis of chiral 4-aryl- $\delta$-lactones. Heterocycles 2010, 80 , 157-162.

(16) Korenaga, T.; Sasaki, R.; Takemoto, T.; Yasuda, T.; Watanabe, M., Computationally-Led Ligand Modification using Interplay between Theory and Experiments: Highly Active Chiral Rhodium Catalyst Controlled by Electronic Effects and $\mathrm{CH}-\pi$ Interactions. Adv. Synth. Catal. 2018, 360, 322-333.

(17) (a) Mino, T.; Miura, K.; Taguchi, H.; Watanabe, K.; Sakamoto, M., BICMAP-rhodium(I)-catalyzed asymmetric 1,4addition of arylboronic acids to coumarins. Tetrahedron: Asymmetry 2015, 26, 1065-1068; (b) Mino, T.; Watanabe, K.; Akiyama, T.; Mizutani, Y.; Miura, K.; Hashimoto, M.; Yoshida, Y.; Sakamoto, M., The second-generation synthesis of BICMAP analogues. Tetrahedron 2018, 74, 3871-3878.

(18) Yu, X.-Q.; Shirai, T.; Yamamoto, Y.; Miyaura, N., Rhodium-catalyzed 1,4-addition of lithium 2-furyltriolborates to unsaturated ketones and esters for enantioselective synthesis of $\gamma$-oxocarboxylic acids by oxidation of the furyl ring with ozone. Chem. Asian J. 2011, 6, 932-937.

(19) Gilbert, S. H.; Fuentes, J. A.; Cordes, D. B.; Slawin, A. M Z.; Clarke, M. L., Phospholane-Phosphite Ligands for Rh Catalyzed Enantioselective Conjugate Addition: Unusually Reactive Catalysts for Challenging Couplings. Eur. J. Org. Chem. 2020, 2020, 30713076.

(20) Defieber, C.; Paquin, J.-F.; Serna, S.; Carreira, E. M., Chiral [2.2.2] Dienes as Ligands for $\mathrm{Rh}(\mathrm{I})$ in Conjugate Additions of Boronic Acids to a Wide Range of Acceptors. Org. Lett. 2004, 6, 3873-3876.

(21) Luo, Y.; Carnell, A. J., Chemoenzymatic Synthesis and Application of Bicyclo[2.2.2]octadiene Ligands: Increased Efficiency in Rhodium-Catalyzed Asymmetric Conjugate Additions by Electronic Tuning. Angew. Chem., Int. Ed. 2010, 49, 2750-2754.

(22) Lu, X.; Lin, S., Pd(II)-Bipyridine Catalyzed Conjugate Addition of Arylboronic Acid to $\alpha, \beta$-Unsaturated Carbonyl Compounds. J. Org. Chem. 2005, 70, 9651-9653.

(23) Ibrahem, I.; Ma, G.; Afewerki, S.; Cordova, A., Palladium/Chiral Amine Co-catalyzed Enantioselective $\beta$-Arylation of $\alpha, \beta$-Unsaturated Aldehydes. Angew. Chem., Int. Ed. 2013, 52, 878882 .

(24) Huh, J. R.; Englund, E. E.; Wang, H.; Huang, R.; Huang, P.; Rastinejad, F.; Inglese, J.; Austin, C. P.; Johnson, R. L.; Huang, W.; Littman, D. R., Identification of Potent and Selective Diphenylpropanamide ROR $\gamma$ Inhibitors. ACS Med. Chem. Lett. 2013, 4, 79-84. 